\title{
Effect of early oseltamivir on outpatients without hypoxia with suspected COVID-19
}

\author{
Satoru Chiba
}

Received: 10 August 2020 / Accepted: 16 November 2020 / Published online: 9 December 2020

(C) Springer-Verlag GmbH Austria, part of Springer Nature 2020

\begin{abstract}
Summary
Background Since December 2019 coronavirus disease 2019 (COVID-19) outbreaks have occurred in China and many countries around the world. The effectiveness of oseltamivir on COVID-19 suspected outpatients without hypoxia was evaluated.

Methods A total of 16 COVID-19 suspected medical personnel and their cohabitation families without hypoxia, who came to the adult fever clinic from March to June 2020 were studied. All patients received antiviral therapy (oseltamivir) and antibacterial therapy together.

Results Most of the infected patients were female (10, $63 \%), 1$ had diabetes (6\%) and 3 hypertension (19\%). The median age was 45 years (interquartile range [IQR] 31-60 years). Oseltamivir administration made the temperature fall within $24 \mathrm{~h}$ in part $(10,63 \%)$. Clinical data were compared between patients receiving early treatment (ET) with oseltamivir, initiated within $24 \mathrm{~h}$, and patients administered late treatment (LT), initiated after this time point. Duration of fever was shorter in the ET group than in the LT group $(31 \pm 21 \mathrm{~h}$ versus $94 \pm 38 \mathrm{~h} ; p<0.001)$. The time from fever onset to treatment initiation correlated with duration of fever $(\mathrm{r}=0.77 ; p<0.001)$ and the time from peak to decline $(\mathrm{r}=0.58 ; p<0.05)$.

Conclusion These findings suggest that early oseltamivir administration may lower the duration of fever in COVID-19 suspected outpatients without hypoxia when it is used in combination with antibacterial therapy.
\end{abstract}

\footnotetext{
S. Chiba, MD, PhD ( $\bowtie)$

Department of Internal Medicine, Sapporo Suzuki

Hospital, 1-27, Shinkotoni-3jo-1chome, Hokkaido, Kita-ku,

Sapporo 001-0903, Japan

chibas_0317@yahoo.co.jp
}

Keywords COVID-19 - Antiviral agents · Early treatment · Duration of fever · Levofloxacin

\section{Introduction}

In late December 2019, an outbreak of an emerging coronavirus disease 2019 (COVID-19) due to severe acute respiratory syndrome coronavirus 2 (SARSCoV-2) started in Wuhan, China and rapidly spread in China and outside [1, 2]. The Governor of Hokkaido declared a state of emergency on 28 February 2020 and people started to avoid going out. The World Health Organization (WHO) declared the epidemic of COVID-19 as a pandemic on 12 March 2020 [3]. The Prime Minister declared a state of emergency on 16 April 2020 in Japan.

According to the recent Chinese study, about $80 \%$ of patients present with mild disease and the overall case fatality rate is about $2.3 \%$ but reaches $8.0 \%$ in patients aged $70-79$ years and $14.8 \%$ in those aged $>80$ years [4]; however, there are probably an important number of asymptomatic carriers in the population and some cases of mild disease will change to severe disease later.

Unfortunately, there are no vaccines approved for COVID-19. Several drugs such as chloroquine, remdesivir, and favipiravir are currently undergoing clinical studies to test their efficacy and safety in the treatment of COVID-19 [5]. A study has revealed that the antivirals to treat influenza (umifenovir, known as Arbidol) can effectively inhibit SARS-CoV-2 infections in vitro [6]. Oseltamivir has been most widely used for influenza, with >65 million treatment courses prescribed worldwide. Oseltamivir was also used as an anti-MERS-CoV therapy in Korea and an anti-SARSCoV-2 therapy in China [7, 8].

This study tested the hypothesis that oseltamivir administration may lower the duration of symptoms, 


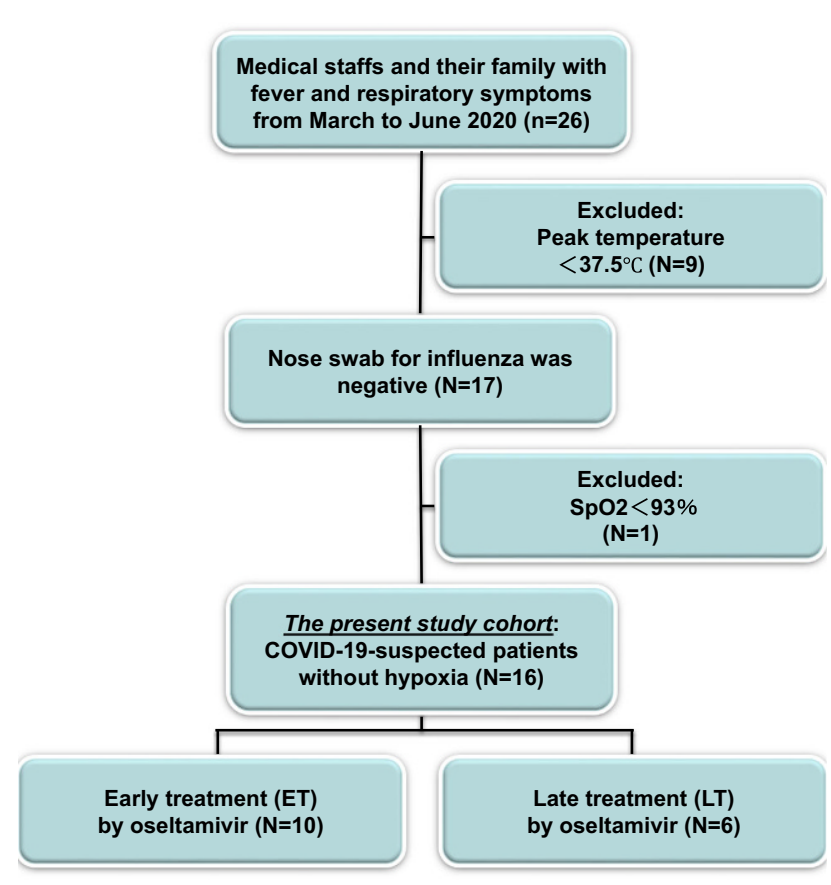

Fig. 1 Flow chart of the COVID-19-suspected outpatients study cohort. SpO2 peripheral capillary oxygen saturation

such as fever, in outpatients with suspected COVID19.

\section{Methods}

\section{Study design and participants}

A total of 26 medical personnel and their cohabitation families with medication in the fever clinic, from 1 March 2020 to 19 June 2020 were prospectively enrolled in the study if they fulfilled five primary criteria: i) age $\geqq 20$ years, ii) peak temperature $\geqq 37.5^{\circ} \mathrm{C}$, iii) one or more respiratory symptoms, iv) a nose swab for influenza A and B was negative and v) peripheral capillary oxygen saturation $(\mathrm{SpO} 2) \geqq 93 \%$ (Fig. 1). Nine with low grade fever (peak temperature $<37.5^{\circ} \mathrm{C}$ ) were excluded. One patient with hypoxia was excluded. Thus, the study population consisted of 16 patients without low grade fever and hypoxia. Eligible participants were given oseltamivir $75 \mathrm{mg}$ orally twice a day for 5 days.

\section{Case definition and variable measurement}

A suspected case of COVID-19 was defined as an individual who had an influenza-like illness, as shown by peak temperature $\geqq 37.5^{\circ} \mathrm{C}$, one or more respiratory symptoms (cough, sputum, or sore throat), and a nose swab for influenza was negative.

Oseltamivir therapy was considered as early treatment (ET) if the patients received treatment within $24 \mathrm{~h}$ of the onset of fever $\left(\geq 37.0^{\circ} \mathrm{C}\right)$ and late treatment (LT) if the antiviral therapy commenced $24 \mathrm{~h}$ after onset of fever.
Duration of fever was defined as the time from the onset of fever $\left(\geqq 37.0^{\circ} \mathrm{C}\right)$ to the decline of fever $\left(<37.0^{\circ} \mathrm{C}\right)$. Time from fever onset to treatment initiation was defined as the interval between onset of fever and the initial administration of oseltamivir. Time from onset to peak was defined as the interval between onset of fever and peak of fever. Time from peak to decline was defined as the interval between peak of fever and decline of fever. Finally, given practical considerations, participants were asked to record data on a diary card twice or more daily in this study.

Breastfeeding and pregnant patients were excluded based on their declaration and pregnancy test results when required. Influenza antigen tests were performed immediately after sample collection. This antigen test ImunoAce Flu is licensed for the diagnosis of human influenza A and B (Tauns Laboratories, Shizuoka, Japan) and has a sensitivity of $67 \%$ [9].

\section{Statistical analysis}

Data are expressed as mean \pm standard deviation or percentages of patients. Differences were compared by Wilcoxon non-parametric test for Gaussian variable. The ratio between baseline and after medication was compared by $\chi^{2}$-test. $p<0.05$ was considered significant for all tests.

\section{Results}

\section{Patients characteristics}

We enrolled 16 patients meeting the inclusion criteria in this study who had at least 7 days of follow-up at the time of the present analysis. The baseline characteristics of the whole population are shown in Table 1. Overall, 10 patients were female $(63 \%)$, with a mean age of 42 years. Median age was 45 years (interquartile range [IQR] 31-60 years). All participants were negative with a local diagnostic test for influenza, of which $16(100 \%)$ were using rapid antigen assays. Less than half had underlying diseases $(5,31 \%)$, including diabetes $(1,6 \%)$ and hypertension $(3,19 \%)$. None of the patients had cardiovascular diseases, lung diseases, chronic kidney diseases or cancer. The influenza was not spreading during the study in Sapporo and the all number of influenza patient reports per fixed point of the coverage period was almost less than 1.00, although the number was 1.61 only in the period from 9 March 2020 to 15 March 2020. The number of people who have taken PCR tests for SARS-CoV-2 was limited in Japan, but the number of people infected with COVID-19 has reached 700 in Sapporo on June 2020. There was the COVID-19 warning announced on 19 June 2020 in Sapporo. 
Table 1 Patient characteristics

\begin{tabular}{|l|l|}
\hline Variables & $\mathbf{n = 1 6}$ \\
\hline Age, years & $42 \pm 16$ \\
\hline Sex & \\
\hline Female, $n$ (\%) & $10(63)$ \\
\hline BMI, kg/m ${ }^{2}$ & $22 \pm 3$ \\
\hline Sp02, \% & $98 \pm 1$ \\
\hline Smoking, $n$ (\%) & $8(50)$ \\
\hline Influenza vaccine, $n$ (\%) & $9(56)$ \\
\hline BCG, $n$ (\%) & $15(94)$ \\
\hline Underlying disease & \\
\hline Hypertension, $n$ (\%) & $3(19)$ \\
\hline Diabetes mellitus, $n$ (\%) & $1(6)$ \\
\hline Dyslipidemia, $n$ (\%) & $3(19)$ \\
\hline Hyperuricemia, $n(\%)$ & $2(13)$ \\
\hline Medications & \\
\hline Oseltamivir, $n$ (\%) & $16(100)$ \\
\hline Levofloxacin, $n(\%)$ & $14(88)$ \\
\hline Garenoxacin, $n(\%)$ & $1(6)$ \\
\hline Amoxicillin/clavulanic acid, $n$ (\%) & $1(6)$ \\
\hline Acetaminophen, $n$ (\%) & $4(25)$ \\
\hline Probiotics (MIYA-BM), $n$ (\%) & $4(25)$ \\
\hline $\begin{array}{l}\text { BMI body mass index, Sp02 peripheral capillary oxygen saturation, } \\
\text { BCG Bacille de Calmette et Guérin, MIYA-BM the probiotic drug that } \\
\text { Contains Clostridium butyricum MIYAIRI 588 }\end{array}$ \\
\hline
\end{tabular}

Table 2 COVID-19-suspected signs and symptoms at baseline and after oseltamivir treatment

\begin{tabular}{|l|l|l|l|}
\hline & Baseline & 7 days & $P$ value \\
\hline Fever, $n$ (\%) & $16(100)$ & $0(0)$ & $<0.001$ \\
\hline Myalgia or fatigue, $n$ (\%) & $13(81)$ & $1(6)$ & $<0.001$ \\
\hline Sore throat, $n$ (\%) & $13(81)$ & $2(13)$ & $<0.001$ \\
\hline Cough, $n$ (\%) & $11(69)$ & $3(19)$ & $<0.001$ \\
\hline Chills, $n$ (\%) & $6(38)$ & $0(0)$ & $<0.01$ \\
\hline Headache, $n$ (\%) & $6(38)$ & $0(0)$ & $<0.01$ \\
\hline Diarrhea, $n$ (\%) & $4(25)$ & $2(13)$ & 0.33 \\
\hline Sputum production, $n(\%)$ & $3(19)$ & $3(19)$ & 1.00 \\
\hline Dyspnea, $n$ (\%) & $2(13)$ & $0(0)$ & 0.16 \\
\hline Appetite loss, $n$ (\%) & $2(13)$ & $1(6)$ & 0.58 \\
\hline Nausea or vomiting, $n(\%)$ & $1(6)$ & $0(0)$ & 0.33 \\
\hline
\end{tabular}

\section{Effect of oseltamivir}

A total of 16 patients received oseltamivir. Among oseltamivir-treated patients, all patients also received antibacterial agents: levofloxacin $(500 \mathrm{mg}$ on day 1 followed by $500 \mathrm{mg}$ per day for the next 6 days), garenoxacin ( $400 \mathrm{mg}$ on day 1 followed by $400 \mathrm{mg}$ per day for the next 6 days), or amoxicillin/clavulanic acid (750 mg on day 1 followed by $750 \mathrm{mg}$ per day for the next 6 days), considering the possibility of bacterial coinfection.

The symptoms of baseline and after oseltamivir treatment are shown in Table 2. Common symptoms at onset of illness were fever $(16,100 \%)$, myalgia and fatigue $(13,81 \%)$, sore throat $(13,81 \%)$, and cough (11,
$69 \%)$; less common symptoms were chills (6, 38\%), headache $(6,38 \%)$, diarrhea $(4,25 \%)$, and sputum production $(3,19 \%)$. None of the patients had olfactory or taste disorders. Oseltamivir helped improve not only fever but also other symptoms in this study.

All patients received oseltamivir, which made the temperature fall to normal $\left(<37.0^{\circ} \mathrm{C}\right)$ within $24 \mathrm{~h}(10$ out of 16 patients, $63 \%)$ and $48 \mathrm{~h}(14,88 \%)$ in part. The fever disappeared in all patients at 4 days after the initial administration of oseltamivir. We let 14 medical personnel wear a mask and come back, because their symptoms disappeared at 7 days. Of these 14 medical personnel 1 had a recurrence of the fever, but the chest computed tomography (CT) did not show pneumonia. Two medical staff took a day off over 1 week, because one had diarrhea and appetite loss, and another had a constant cough. Their chest CT did not show any pneumonia.

The study was divided into two groups if the patients received treatment at $24 \mathrm{~h}$ of the fever onset: 10 patients $(63 \%)$ received oseltamivir within $24 \mathrm{~h}$ of the onset of fever (early treatment: ET) and 6 after $24 \mathrm{~h}$ (late treatment: LT). In the ET and the LT groups two patients each received acetaminophen. Some patients' fever has gone within $24 \mathrm{~h}(6,60 \%$ versus 4 , $67 \%, p=\mathrm{NS})$ and $48 \mathrm{~h}(9,90 \%$ versus $5,83 \%, p=\mathrm{NS})$ after the initial administration in the ET and the LT groups, respectively. The two groups were comparable in terms of peak temperature, duration of fever, the time from onset to peak, and the time from peak to decline. Duration of fever were significantly shorter in the ET group than in the LT group $(31 \pm 21 \mathrm{~h}$ versus $94 \pm 38$ h; $P<0.001$, Fig. 2a). The time from peak to decline was significantly shorter in ET than LT $(24 \pm 24$ versus $70 \pm 50 \mathrm{~h} ; p<0.05$ ) (Fig. $2 \mathrm{c}$ ). The time from onset to peak was also shorter in ET than LT $(7 \pm 8 \mathrm{~h}$ versus $24 \pm 16 \mathrm{~h}$; $p<0.05$, Fig. $2 \mathrm{~b}$ ). A comparison of the peak temperature showed no significant difference between the ET and the LT groups $\left(38.1 \pm 0.6^{\circ} \mathrm{C}\right.$ versus $38.0 \pm 0.9^{\circ} \mathrm{C} ; p=0.80$ ).

\section{Interaction between duration of fever and time from fever onset to treatment initiation}

Univariate regression analysis confirmed that the time from fever onset to treatment initiation positively correlated with the duration of fever $(\mathrm{r}=0.767, p<0.001$, Fig. 3a). Likewise, the significant relationship was observed between the time from fever onset to treatment initiation and the time from peak to decline $(\mathrm{r}=0.583$, $p<0.05$, Fig. $3 b$ ). The time from onset to peak correlated with the time from fever onset to treatment initiation $(\mathrm{r}=0.562, p<0.05)$. The peak temperature did not correlate with the time from fever onset to treatment initiation $(\mathrm{r}=-0.063, p=\mathrm{NS})$. 

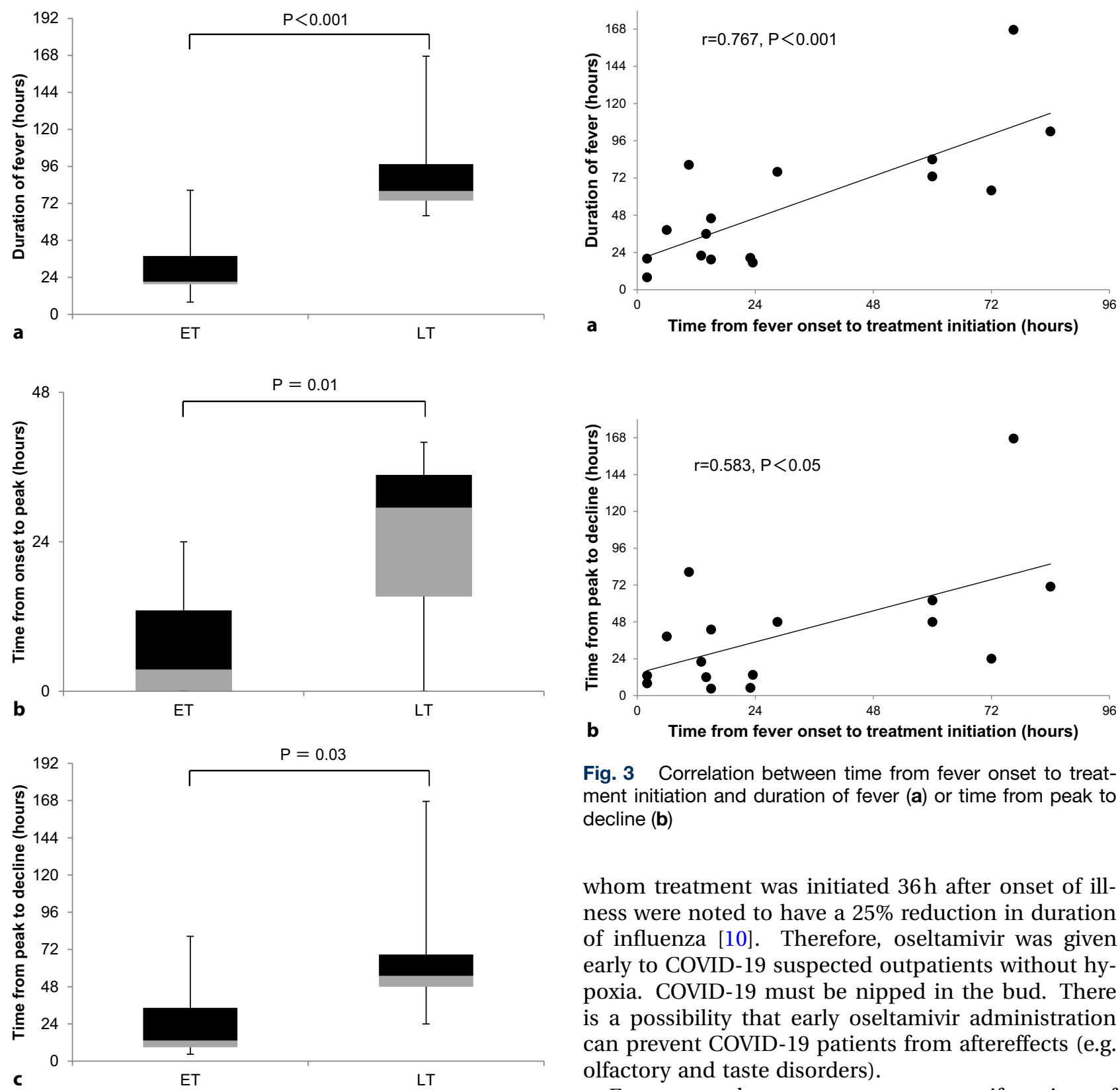

Fig. 3 Correlation between time from fever onset to treatment initiation and duration of fever (a) or time from peak to decline (b)

whom treatment was initiated $36 \mathrm{~h}$ after onset of illness were noted to have a $25 \%$ reduction in duration of influenza [10]. Therefore, oseltamivir was given early to COVID-19 suspected outpatients without hypoxia. COVID-19 must be nipped in the bud. There is a possibility that early oseltamivir administration can prevent COVID-19 patients from aftereffects (e.g. olfactory and taste disorders).

Fever was the most common manifestation of

Fig. 2 Comparison of ET $(n=10)$ versus LT $(n=6)$. a Duration of fever, $\mathbf{b}$ time from onset to peak, $\mathbf{c}$ time from peak to decline. $E T$ patients receiving early treatment with oseltamivir, initiated within $24 \mathrm{~h}, L T$ patients receiving late treatment with oseltamivir, initiated after $24 \mathrm{~h}$

\section{Discussion}

Oseltamivir was not effective enough as empirical treatment in COVID-19 infections with dyspnea or hypoxia in Wuhan [8]. COVID-19 pneumonia may be resistant at the beginning of treatment. The treatment must be initiated before COVID-19 pneumonia appears. Oseltamivir was licensed for use within $48 \mathrm{~h}$ of onset of the symptoms, in influenza patients. Early initiation of oseltamivir after onset of symptoms increases its therapeutic effects, although even those in COVID-19 followed by respiratory symptoms [8]. In a disease such as influenza that typically resolves within 3-4 days, using twice daily assessments may be insensitive to detect rapid improvement but COVID19 sometimes differs from influenza in longer duration of fever. The study by Chen et al. showed that the median duration of fever in the COVID-19 patients not in intensive care units (ICU) was 9 days [11]. All patients in this study received oseltamivir within 4 days after fever onset. In more than half the patients, the fever has gone within $24 \mathrm{~h}$ after the initial administration. It has shown that early oseltamivir administration can reduce the duration of fever and the time from peak to decline in the COVID-19 suspected outpatients without hypoxia when it is used in combination with antibacterial therapy. The presence of fever may select for a population with a greater 
likelihood to benefit from oseltamivir. Although oseltamivir possibly causes hypothermia through reduction of sympathetic nerve activity [12], it helped improve other symptoms than fever in this study.

Paracetamol (acetaminophen) is recommended as the first line of antipyretic for anti-inflammatory effects in COVID-19 patients [13]. In the absence of any FDA-approved drugs for treatment of COVID19, many clinicians have turned to existing drugs to prevent or treat the disease. Some drugs that have been tried based on hypotheses or limited evidence include chloroquine, remdesivir, favipiravir, and neuraminidase inhibitors used to treat influenza [5]. In addition, many adjunctive therapies including vitamin $\mathrm{C}$ are used as supportive care for COVID19 patients. Vitamin $C$ appears to be effective as an antiviral agent, especially against influenza viruses [13]. Serious adverse events were reported in chloroquine, remdesivir, and favipiravir but these drugs are rather hard to obtain in our hospital. Neuraminidase inhibitors (e.g., oseltamivir) are not expected to be effective for prevention or treatment of COVID-19, because SARS-CoV-2 does not contain neuraminidase [14] but oral oseltamivir has been widely used for COVID-19 or suspected cases in Chinese hospitals [8]. The trials of oseltamivir are being established in China and oseltamivir treatment was safe with mild side effects [5]. The study by Coenen et al. showed that primary care patients with influenza-like illness testing positive for coronavirus (not including SARS$\mathrm{CoV}-2$ ) recovered sooner when oseltamivir was added to usual care compared with usual care alone [15]. Therefore, the hypothesis that oseltamivir administration may lower the duration of symptoms, such as fever in COVID-19 suspected patients was tested. Oseltamivir really helped improve the symptoms including fever, fatigue, sore throat, and cough in this study (Table 2). It may be preferable that vitamin $\mathrm{C}$ and/or paracetamol are used with oseltamivir in the future.

Chinese medicine is a promising adjuvant therapy for infectious disease in light of its safety and pleiotropic effect of anti-inflammation, etc. Besides its use as spice in cuisine, star anise is one of the vital ingredients of the Chinese medicinal herbs and is widely known for its antiviral effects. It is also the source of the precursor molecule, shikimic acid, which is used in the manufacture of oseltamivir, an antiviral medication for influenza [16]. It was therefore thought that oseltamivir would be effective in the treatment of COVID-19 and oseltamivir was used in combination with antibacterial therapy, because previous studies have shown that patients with COVID19 can have secondary infections with bacteria [17]. Many influenza patients in the $1918 \mathrm{flu}$ pandemic also had secondary bacterial pneumonia [18]. In addition to their antimicrobial activity, fluoroquinolones, such as levofloxacin exert anti-inflammatory activity [19]. In the absence of specific antiviral drugs been proven against SARS-CoV-2, antibiotics and/or acetaminophen may together be used with oseltamivir. Despite its small sample size, this study showed that oseltamivir treatment is significantly associated with decline of fever/viral disappearance in COVID-19 suspected patients and its effect is reinforced by antibacterial therapy. Medical staff are placed in a high-risk situation and early oseltamivir administration may prevent COVID-19 infected pneumonia and enable medical staff to return to work as soon as possible.

\section{Limitations of the study}

This study has some limitations; however, in the current context, it is believed that the results should be shared with the scientific community. First, the number of patients was too small to draw definite conclusions. Further studies are clearly needed. Second, there was no control group for patients without use of oseltamivir, therefore, it is difficult to directly compare the duration of fever in patients with and without use of oseltamivir. Third, there was a lack of coronavirus tests in the population because in Japan the test for SARS-CoV-2 could only be performed if pneumonia was present or a confirmed close contact with a SARSCoV-2 patient in the beginning. The COVID-19 pandemic was going around during the study in Sapporo and many people could have been unknowingly positive for SARS-CoV-2. Fourth, a definitive diagnosis of influenza requires a PCR-based method in general. Therefore, this study may have included patients with influenza-like illness or other respiratory diseases. Influenza diagnosis information was obtained based on use of rapid diagnosis kits by medical institution and the sensitivity of the influenza antigen test is equal to that of the PCR test for SARS-CoV-2. Therefore, it is believed that the diagnosis information was reliable.

Acknowledgements The author thanks all of the staff members and their cohabitation families of the Sapporo Suzuki Hospital who agreed to participate in this study, the director, the chairman, the assistant director, the other doctors and all the nurses who cared for these patients and all the technical and paramedical staff of the hospitalization units for their support in this difficult context.

\section{Compliance with ethical guidelines}

Conflict of interest S. Chiba declares that he has no competing interests.

Ethical standards This study was approved by the Ethics in Research Committee of Sapporo Suzuki Hospital (No. 2020001, 14 February 2020) and the procedures were in accordance with institutional guidelines. Verbal informed consent was obtained from each study patient. Before analyzing the data all identifiable information was deleted and specific individuals cannot be identified by these data. Therefore, this was approved by the ethics committee. 


\section{References}

1. Lai CC, Shih TP, Ko WC, Tang HJ, Hsueh PR. Severe acute respiratory syndrome coronavirus 2 (SARS-CoV-2) and coronavirus disease-2019 (COVID-19): the epidemic and the challenges. Int JAntimicrob Agents. 2020;55:105924.

2. Wang LS, Wang YR, Ye DW, Liu QQ. Areview of the 2019 Novel Coronavirus (COVID-19) based on current evidence. Int J Antimicrob Agents. 2020;19:105948.

3. WHO. Director-General's opening remarks at the media briefing on COVID-19-11 March. 2020. https://www. who.int/director-general/speeches/detail/who-directorgeneral-s-opening-remarks-at-the-media-briefing-oncovid-19--11-march-2020.

4. Wu Z, McGoogan JM. Characteristics of and important lessons from the coronavirus disease 2019 (COVID-19) outbreakin China: summary of a report of 72314 cases from the Chinese Center for Disease Control and Prevention. JAMA. 2020;323(13):1239-42.

5. Guangdi L, De Clercq E. Therapeutic options for the 2019 novel coronavirus (2019-nCoV). Nat Rev Drug Discov. 2020;19(3):149-50.

6. Khamitov RA, Loginova SLA, Shchukina VN, Borisevich SV, Maksimov VA, Shuster AM. Antiviral activity of arbidol and its derivatives against the pathogen of severe acute respiratory syndrome in the cell cultures [in Russian. Vopr Virusol. 2008;53(4):9-13.

7. Al-Tawfiq JA, Rabaan AA, Hinedi K. Influenza is more common than Middle East Respiratory Syndrome Coronavirus (MERS-CoV) among hospitalized adult Saudi patients. Travel Med Infect Dis. 2017;20:56-60.

8. Wang D, Hu B, Hu C, Zhu F, Liu X, Zhang J, et al. Clinical characteristics of 138 hospitalized patients with 2019 novel Coronavirus-infected pneumonia in Wuhan, China. JAMA. 2020;323(11):1061-9.

9. Yamanaka T, Nemoto M, Bannai H, Tsujimura K, Kondo T, Matsumura T, et al. Evaluation of twenty-two rapid antigen detection tests in the diagnosis of Equine Influenza caused by viruses of H3N8 subtype. Influenza Other Respir Viruses. 2016;10:127-33.

10. IMPACT Study Group, Aoki FY, Macleod MD, Paggiaro P, et al. Early administration of oral oseltamivir increases the benefits of influenza treatment. J Antimicrob Chemother. 2003;51:123-9.

11. Chen J, Qi T, Liu L, Ling Y, Qian Z, Li T, et al. Clinical progression of patients with COVID-19 in Shanghai, China. JInfect. 2020;80(5):el-e6.

12. Ono H, Iwajima Y, Nagano Y, Chazono K, Maeda Y, Ohsawa $\mathrm{M}$, et al. Reduction in sympathetic nerve activity as a possible mechanism for the hypothermic effect of oseltamivir, an anti-influenza virus drug, in normal mice. Basic Clin Pharmacol Toxicol. 2013;113(1):25-30.

13. Kakodkar P, Kaka N, Baig MN. A comprehensive literature review on the clinical presentation, and management of the pandemic Coronavirus disease 2019 (COVID-19). Cureus. 2020;12(4):e7560.

14. Some Drugs for COVID-19, Med Lett Drugs Ther 2020 Apr 6;62(1595):49-50.

15. Coenen S, van der Velden AW, Cianci D, Goossens H, Bongard E, Saville BR, et al. Oseltamivir for coronavirus illness: post-hoc exploratory analysis of an open-label, pragmatic, randomised controlled trial in European primary care from 2016 to 2018. BrJGen Pract. 2020;70(696):e444-e9.

16. Patra JK, Das G, Bose S, Banerjee S, Vishnuprasad CN, Del Pilar Rodriguez-Torres M, et al. Star anise (Illicium verum): chemical compounds, antiviral properties, and clinical relevance. Phytother Res. 2020;34(6):1248-67.

17. Chen N, Zhou M, Dong X, Qu J, Gong F, Han Y, et al. Epidemiological and clinical characteristics of 99 cases of 2019 novel coronavirus pneumonia in Wuhan, China: a descriptive study. Lancet. 2020;395(10223):507-13.

18. Sheng ZM, Chertow DS, Ambroggio X, McCall S, Przygodzki RM, Cunningham RE, etal. Autopsyseries of 68 cases dying before and during the 1918 influenza pandemic peak. Proc Natl Acad Sci U SA. 2011;108(39):16416-21.

19. Zusso M, LunardiV, FranceschiniD, PagettaA, Lo R, StifaniS, et al. Ciprofloxacin and levofloxacin attenuate microglia inflammatory response via TLR4/NF-kB pathway. J Neuroinflammation. 2019;16(1):148.

Publisher's Note Springer Nature remains neutral with regard to jurisdictional claims in published maps and institutional affiliations. 